\title{
Improving TCP Performance based on Fuzzy-Logic for Mobile Ad hoc Networks
}

\author{
Ibrahim K. Tabash \\ Department of Computer \\ Engineering \\ Aligarh Muslim University \\ (AMU), Aligarh-202002, UP \\ India.
}

\author{
M. Salim Beg \\ Department of Electronics \\ Engineering \\ Aligarh Muslim University \\ (AMU), Aligarh-202002, UP \\ India.
}

\author{
Nesar Ahmad \\ Department of Computer \\ Engineering \\ Aligarh Muslim University \\ (AMU), Aligarh-202002, UP \\ India.
}

\begin{abstract}
Transmission Control Protocol (TCP) is designed for wired networks and the sender assumes that packet loss is an indicator of network congestion. However, this assumption may not apply to Mobile Ad hoc Networks (MANETs). Numerous transmission control protocols have been proposed in the literature to address the problem of congestion in MANETs. Their performance is highly dependent on networks conditions, probe and explicit notification messages. This paper proposed a fuzzy logic based inference system based on the factors of expected throughput and actual throughput to dynamically adjust the congestion window size to improve the performance of TCP in MANETs. Fuzzy inference system is an attractive approach used in MANETs which suffer from all kinds of uncertainty, randomness and vagueness. The scheme does not rely on any explicit feedback from the network and requires only sender side modifications. The simulation study examine the throughput, fairness index degree, packet loss percentage and packet delay as performance metrics of the ad hoc network with regard to equal sharing of network bandwidth among multiple TCP flows. The results observe that the proposed scheme achieves the desired goals of performance improvement in terms of packet loss and packet delay compared to TCP-Vegas, Reno and New Reno.
\end{abstract}

\section{Keywords}

TCP, Fuzzy Logic, MANETs.

\section{INTRODUCTION}

Mobile Ad hoc Networks (MANETs) are a collection of mobile nodes forming a dynamic autonomous network. Nodes communicate with each other without the intervention of centralized access points or base stations. In such a network, each node acts both as a router and as a host. MANETs are getting extremely popular with the advent of various types of mobile devices. However, rapidly changing connectivity, network partitions, higher error rates, security threats, frequent collision probability, bandwidth and power constraints together pose new problems in designing protocols [1].

Several research studies have been conducted concerning the performance of transmission control protocol (TCP) in MANETs employing IEEE 802.11 protocol [2]. The performance of TCP in MANETs is not satisfactory. Lossy wireless channels, inefficient medium access control (MAC) protocols, large overhead of routing protocols, and interaction among different protocol layers are all responsible for the poor performance.
This paper proposed a novel idea that uses a fuzzy logic-based inference system to dynamically control the congestion window size according to the network conditions by observing the expected throughput, actual throughput, and focus on issues related to reliability of data transport over MANETs. A fuzzy logic-based approach is unlike other techniques proposed in the past.

The structure of this paper is as follows. Section 2 discusses related works. Section 3 presents an overview of fuzzy logic control. In section 4, we present our proposed protocol. Section 5 focuses on the simulation environment and performance metrics. Section 6 deals with the discussion and analysis of simulation results. Finally, section 7 concludes and highlights the findings of the paper.

\section{RELATED WORKS}

In recent years, several approaches have been proposed to improve TCP performance at the transport layer 3-9, as shown in Table I.

Table I. Classification of the existing proposals

\begin{tabular}{|c|c|c|c|}
\hline \multicolumn{3}{|c|}{ Proposals for TCP over MANETs } \\
\hline Network Dependent & \multicolumn{2}{|c|}{ Network Independent } \\
\hline \begin{tabular}{c|c|c|} 
TCP \\
Variants
\end{tabular} & $\begin{array}{c}\text { Non- } \\
\text { TCP }\end{array}$ & $\begin{array}{c}\text { Routing Protocol } \\
\text { Dependent }\end{array}$ & $\begin{array}{c}\text { Fully } \\
\text { Independent }\end{array}$ \\
\hline $\begin{array}{c}\text { TCP-ELFN } \\
{[3]}\end{array}$ & $\begin{array}{c}\text { ATP } \\
{[6]}\end{array}$ & & $\begin{array}{c}\text { Learning-TCP } \\
{[9]}\end{array}$ \\
\cline { 1 - 1 } TCP- \\
$\begin{array}{c}\text { Feedback } \\
{[4]}\end{array}$ & ITP & FuzzyTCP [8] & \\
\cline { 1 - 1 } $\begin{array}{c}\text { Ad hoc TCP } \\
{[5]}\end{array}$ & & & $\begin{array}{c}\text { FL-TCP Our } \\
\text { Work }\end{array}$ \\
\end{tabular}

In the network dependent protocols [3-7], explicit link failure notifications are used to freeze TCP state upon the occurrence of a route failure. These proposals are network dependent as they rely on feedback information from the network such as congestion, link failure, and available bandwidth notifications. But these proposals may perform poorly when the feedback information from network is unavailable or unreliable. On the other hand, network independent protocols [8, 9] do not require any feedback from the network. Instead, they perform the loss classification and take appropriate actions by observing various parameters such as mean and variance of the round-trip times and hop-length for the TCP connections. 
In [7], the authors propose an improved transport protocol (ITP) in MANETs. ITP uses a rate-based transmission scheme with fuzzy logic control to tune the proper data rate. ITP also gets the MAC layer information and uses a fuzzy logic controller to estimate the appropriate data rate for transmission. Moreover, ITP uses a feedback scheme to adjust data flow by receiving a feedback packet. The difference between traditional TCP and ITP is that ITP adjusts its transmission rate using the received packet instead of the acknowledgments or lost packets.

The proposal in [8] investigates the use of fuzzy logic theory for assisting the TCP error detection mechanism in MANETs. An elementary fuzzy logic engine is presented as an intelligent technique for discriminating packet loss due to congestion from packet loss by wireless induced errors. The architecture of the proposed fuzzy-based error detection mechanism is also introduced and discussed. The full approach, for inferring the internal state of the network relies on Round Trip Time (RTT) measurements.

Some of the recent works are beginning to evaluate the performance of TCP in the context of MANETs [10]. In [11], the authors have investigated the performance of TCP over three different medium access control protocol operating in a multi-hop wireless network. However, a new metric in the form of expected throughput was introduced and used to compare the achieved throughput. In [12], the impact of node mobility was not considered.

In fact, research on efficient transport protocols for MANETs is one of the most active topics in MANET's community. Thus, TCP is affected by node mobility and link errors. There are several proposals that suggest either a new transport protocol or enhancements to the traditional transmission control protocol (TCP) to work efficiently in MANETs.

The proposed FL-TCP presents a new implementation of earlier work [13], incorporating substantial changes to the original FL-TCP proposal. The new proposal introduces: a novel approach to calculate the amount of congestion window size value, a new design of the fuzzy logic controller.

\section{FUZZY-LOGIC CONTROL: OVERVIEW}

Fuzzy logic controllers (FLC), like expert systems, can be used to model human experiences and human decisionmaking behaviors [14]. In FLC the input-output relationship is expressed by using a set of linguistic rules or relational expressions. An FLC basically consists of four important parts including a fuzzifier, a defuzzifier, an inference engine and a rule base. As in many fuzzy control applications, the input data are usually crisp, so fuzzification is necessary to convert the input crisp data into a suitable set of linguistic value that is needed in inference engine. Singleton fuzzifier is the general fuzzification method used to map the crisp input to a Singleton fuzzy set.

In the rule base of an FLC, a set of fuzzy control rules, which characterize the dynamic behavior of system, are defined. The inference engine is used to form inferences and draw conclusions from the fuzzy control rules. Fig. 1, shows the Fuzzy Logic Controller Architecture.

The output of the inference engine is sent to the defuzzification unit. Defuzzification is a mapping from a space of fuzzy control actions into a space of crisp control actions. Suppose the FLC has $n$ input variables including $x_{1}$, $x_{2}, x_{3}, \ldots, x_{n}$. The input vector $X$ is defined as: $X=\left(x_{1}, x_{2}\right.$, $\left.x_{3}, \ldots, x_{n}\right)^{T}$. Furthermore, suppose the rule base consists of $K$ rules with the following general form:

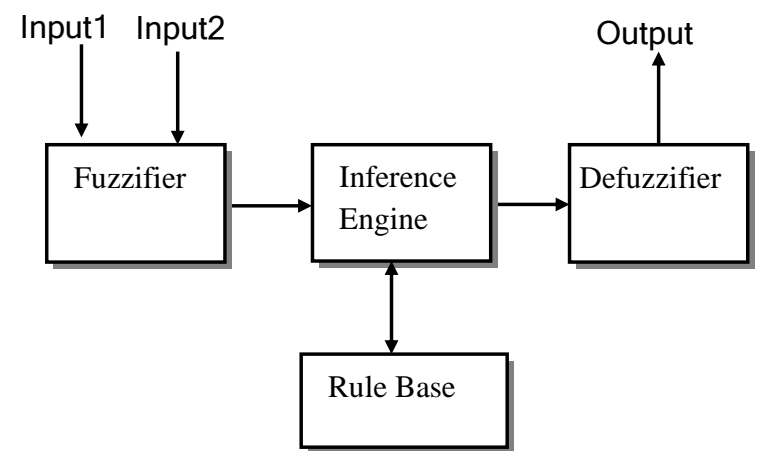

Fig 1: Fuzzy-Logic Controller Architecture

$$
\begin{aligned}
& \text { Rule }-1: \text { if } X=\left(A_{11}, A_{12}, \ldots, A_{1 n}\right), \text { then } y \text { is } B_{1} \\
& \text { Rule }-2: \text { if } X=\left(A_{11}, A_{12}, \ldots, A_{1 n}\right), \text { then } y \text { is } B_{2} \\
& \text { Rule }-k: \text { if } X=\left(A_{11}, A_{12}, \ldots, A_{1 n}\right) \text {, then } y \text { is } B_{k}
\end{aligned}
$$

Where in the $j^{\text {th }}$ rule $A_{i j}$ and $B_{j}$ are fuzzy sets of linguistic variables $x_{1}, x_{2}, x_{3}, \ldots, x_{n}$ and $y$, respectively. The output $f(X)$ of this fuzzy controller with Singleton fuzzifier, product inference engine and center-average defuzzifier can be calculated as [14]:

$$
f(X)=\sum_{j=1}^{K} y_{0}^{j} \prod_{i=1}^{n} \mu_{i}^{j}\left(x_{i}\right) / \sum_{j=1}^{K} \prod_{i=1}^{n} \mu_{i}^{j}\left(x_{i}\right)
$$

Where $y_{0}^{j}$ is the center value of the output fuzzy set $B_{j}$, in the $j^{\text {th }}$ rule. $\mu_{i}^{j}\left(x_{i}\right)$ is the membership function for fuzzy sets. In our proposed model we use two input variables of Fuzzy Controller which present the expected and actual throughput measures, and the output will be the updated probability value for the congestion window.

\section{PROPOSED PROTOCOL: FL-TCP}

The proposed protocol is based on two important factors in order to estimate the network conditions. These two factors are Expected throughput and Actual throughput. The protocol Fuzzy Logic TCP named as (FL-TCP). Here, given a brief introduction to TCP-Vegas protocol [15] to understand the expected and the actual throughputs.

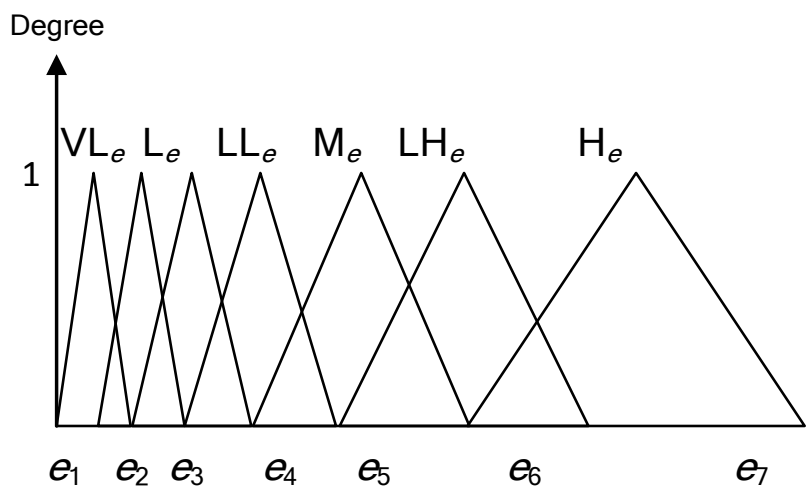

Fig 2: Membership function for Expected Throughput 


\subsection{A brief Introduction to TCP-Vegas}

TCP Vegas uses the difference between expected and actual flow rates to estimate the available bandwidth in the network. The idea is that when the network is not congested, the actual flow rate will be close to the expected flow rate, and otherwise, the actual rate will be smaller than the expected flow rate [15]. Using this difference in flow rates, TCP Vegas estimates the congestion level in the network and updates the window size accordingly. Here, one can notice that the difference in the flow rates can be easily translated into the difference between the window size and the number of acknowledged packets during the Round Trip Time RTT, using the equation:

$$
\text { Diff }=(\text { Expected }- \text { Actual }) * \text { BaseRTT }
$$

Where Expected is the expected rate, Actual is the actual rate, and BaseRTT is the minimum round trip time. The following steps show the details of the TCP-Vegas algorithm:

Step-1: the source computes the expected flow rate Expected $=C W N D /$ BaseRTT, where $C W N D$ is the current window size and BaseRTT is the minimum Round Trip Time.

Step-2: the source estimates the current flow rate by using the actual round trip time according to Actual $=C W N D / R T T$, where $R T T$ is the actual round trip time of a packet.

Step-3: The source, using the expected and actual flow rates, computes the estimated backlog in the queue from equation (2).

Step-4: Based on Diff, the source updates its window size as follows:

$$
C W N D=\left\{\begin{array}{cll}
C W N D+1 & \text { if } & \text { Diff }<\alpha \\
C W N D-1 & \text { if } & \text { Diff }>\beta \\
C W N D & & \text { otherwise }
\end{array}\right.
$$

Where $\alpha, \beta$ are the minimum and the maximum bandwidth thresholds.

\subsection{Details of Proposed Protocol: FL-TCP}

In the proposed FL-TCP protocol, we use a fuzzy logic inference system to determine the difference between expected and actual throughput. However, the idea behind our FL-TCP is not using any fixed thresholds such as $\alpha, \beta$, and dynamically updating the congestion window size by probabilistic value estimated by the fuzzy logic controller. FL-TCP includes the following three steps: setup the fuzzy member, construct the fuzzy rule base to update the congestion window and defuzzification process.

Step-1: Setup the fuzzy member: We have two factors in fuzzy members to consider the status of the MANET, the expected throughput $e$ and actual throughput $a$. We divide the expected throughput and actual throughput into seven functions, the Very Low $\left(\mathrm{VL}_{e}\right)$, Low $\left(\mathrm{L}_{e}\right)$, Little Low $\left(\mathrm{LL}_{e}\right)$, Medium $\left(\mathrm{M}_{e}\right)$, Little High $\left(\mathrm{LH}_{e}\right)$, High $\left(\mathrm{H}_{e}\right)$, and Very High $\left(\mathrm{VH}_{e}\right)$. However, the throughput load needs to be between the $\left[e_{0}, e_{\max }\right]$. We used triangular-shape membership function to represent the continuous throughput state shown in Fig. 2, and actual throughput shown in Fig. 3 .

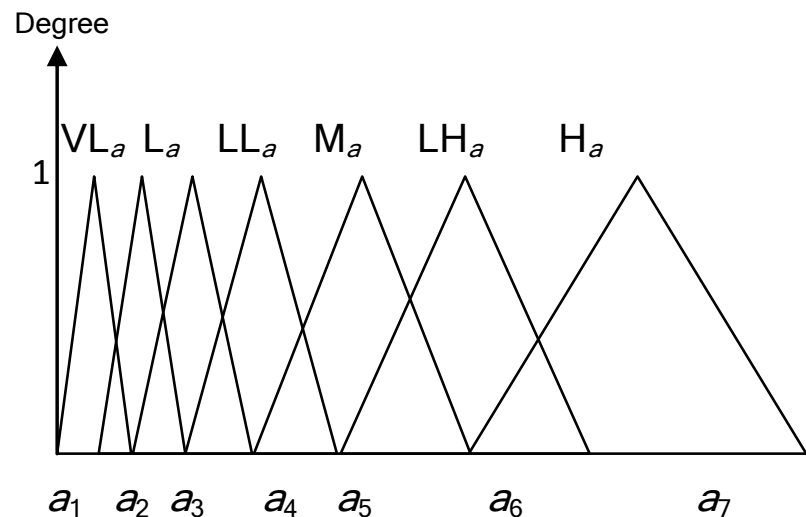

Fig 3: Membership function for Actual Throughput

The congestion window size $(c w)$ is divided into six states: Decrease Very Large $\left(\operatorname{Dec}_{4}\right)$, Decrease Large $\left(\operatorname{Dec}_{3}\right)$, Decrease Medium $\left(\operatorname{Dec}_{2}\right)$, Decrease Small $\left(\operatorname{Dec}_{1}\right)$, No Change (No_C), and Increase (Inc). Similarly, the values need to be between the $\left[\mathrm{Dec}_{4}, \mathrm{Inc}\right]$. We set the present six states between [-2.0, 0.005] as shown in Fig. 4 .

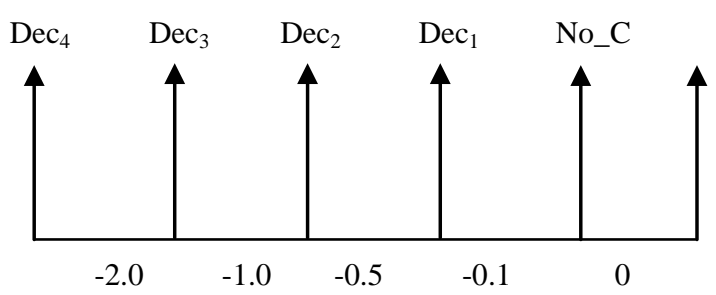

Fig 4: The state of Congestion Window

Step-2: Construct the fuzzy rule-based system to update the congestion window and fuzzy engine: The if-then fuzzy rulebased system can be used to represent the congestion window state. We infer twenty eight rules and discuss these rules one by one in Table II. In fuzzy rule-base system, we do not consider that the rules have actual throughput higher than the expected throughput.

Step-3: defuzzification process: In the Defuzzification component we use the Center of Average to calculate the output value for Fuzzy Controller which presents the updated probability value for the congestion window by using equation (1).

TABLE II. Fuzzy Rule Base System

\begin{tabular}{|c|l|}
\hline \multicolumn{2}{|c|}{ Fuzzy Rule Base System of FL-TCP } \\
\hline Rule 1 & If $e=\mathrm{VL}_{e}$ and $a=\mathrm{VL}_{a}$ then $c w=\mathrm{Inc}$ \\
\hline Rule 2 & If $e=\mathrm{L}_{e}$ and $a=\mathrm{VL}_{a}$ then $c w=\mathrm{No}_{-} \mathrm{C}$ \\
\hline Rule 3 & If $e=\mathrm{L}_{e}$ and $a=\mathrm{L}_{a}$ then $c w=\mathrm{Inc}$ \\
\hline Rule 4 & If $e=\mathrm{LL}_{e}$ and $a=\mathrm{VL}_{a}$ then $c w=\operatorname{Dec}_{1}$ \\
\hline
\end{tabular}




\begin{tabular}{|c|c|}
\hline Rule 5 & If $e=\mathrm{LL}_{e}$ and $a=\mathrm{L}_{a}$ then $c w=$ No_C \\
\hline Rule 6 & If $e=\mathrm{LL}_{e}$ and $a=\mathrm{LL}_{a}$ then $c w=\mathrm{Inc}$ \\
\hline Rule 7 & If $e=\mathrm{M}_{e}$ and $a=\mathrm{VL}_{a}$ then $c w=\mathrm{Dec}_{2}$ \\
\hline Rule 8 & If $e=\mathrm{M}_{e}$ and $a=\mathrm{L}_{a}$ then $c w=\operatorname{Dec}_{1}$ \\
\hline Rule 9 & If $e=\mathrm{M}_{e}$ and $a=\mathrm{LL}_{a}$ then $c w=$ No_C \\
\hline Rule 10 & If $e=\mathrm{M}_{e}$ and $a=\mathrm{M}_{a}$ then $c w=$ Inc \\
\hline Rule 11 & If $e=\mathrm{LH}_{e}$ and $a=\mathrm{VL}_{a}$ then $c w=\operatorname{Dec}_{3}$ \\
\hline Rule 12 & If $e=\mathrm{LH}_{e}$ and $a=\mathrm{L}_{a}$ then $c w=\operatorname{Dec}_{2}$ \\
\hline Rule 13 & If $e=\mathrm{LH}_{e}$ and $a=\mathrm{LL}_{a}$ then $c w=\mathrm{Dec}_{1}$ \\
\hline Rule 14 & If $e=\mathrm{LH}_{\rho}$ and $a=\mathrm{M}_{a}$ then $c w=\mathrm{No} \mathrm{C}$ \\
\hline Rule 15 & If $e=\mathrm{LH}_{e}$ and $a=\mathrm{LH}_{a}$ then $c w=\mathrm{Inc}$ \\
\hline Rule 16 & If $e=\mathrm{H}_{e}$ and $a=\mathrm{VL}_{a}$ then $c w=\mathrm{Dec}_{4}$ \\
\hline Rule 17 & If $e=\mathrm{H}_{e}$ and $a=\mathrm{L}_{a}$ then $c w=\operatorname{Dec}_{3}$ \\
\hline Rule 18 & If $e=\mathrm{H}_{e}$ and $a=\mathrm{LL}_{a}$ then $c w=\operatorname{Dec}_{2}$ \\
\hline Rule 19 & If $e=\mathrm{H}_{e}$ and $a=\mathrm{M}_{a}$ then $c w=\operatorname{Dec}_{1}$ \\
\hline Rule 20 & If $e=\mathrm{H}_{e}$ and $a=\mathrm{LH}_{a}$ then $c w=$ No_C \\
\hline Rule 21 & If $e=\mathrm{H}_{e}$ and $a=\mathrm{H}_{a}$ then $c w=$ Inc \\
\hline Rule 22 & If $e=\mathrm{VH}_{e}$ and $a=\mathrm{VL}_{a}$ then $c w=\mathrm{Dec}_{4}$ \\
\hline Rule 23 & If $e=\mathrm{VH}_{e}$ and $a=\mathrm{L}_{a}$ then $c w=\operatorname{Dec}_{4}$ \\
\hline Rule 24 & If $e=\mathrm{VH}_{e}$ and $a=\mathrm{LL}_{a}$ then $c w=\mathrm{Dec}_{3}$ \\
\hline Rule 25 & If $e=\mathrm{VH}_{e}$ and $a=\mathrm{M}_{a}$ then $c w=\mathrm{Dec}_{2}$ \\
\hline Rule 26 & If $e=\mathrm{VH}_{e}$ and $a=\mathrm{LH}_{a}$ then $c w=\mathrm{Dec}_{1}$ \\
\hline Rule 27 & If $e=\mathrm{VH}_{e}$ and $a=\mathrm{H}_{a}$ then $c w=$ No_C \\
\hline Rule 28 & If $e=\mathrm{VH}_{e}$ and $a=\mathrm{VH}_{a}$ then $c w=\mathrm{Inc}$ \\
\hline
\end{tabular}

\section{SIMULATION ENVIRONMENT AND PERFORMANCE METRICS}

In this section, we present and discuss the simulation environment setting for MANETs over AODV routing protocol, and evaluate the impact of node's mobility. All simulation experiments are carried out using NS-2 [16].

A mobile network consisting of 50 nodes and moving over an area of $1000 \times 1000$ meter has been considered. Table III shows the values of parameters used in this move for mobile scenario. The nodes move according to the Random WayPoint (RWP) mobility model. In this model, each node picks a random destination and speed in the square area and then travels to the destination in a straight line. Once a node arrives at its destination, it pauses, picks another destination, and continues onward. We use a pause time of 2 seconds so that each node before picking another destination pauses for 2 seconds. Also, node mobility average is 0 to $20 \mathrm{~m} / \mathrm{sec}$ in our simulation. In order to evaluate the performance of our proposed FL-TCP, we used multiple concurrent flows: 1, 5, 10 and 20 flows. Each flow session starts at 5 seconds and session ends at 399 seconds. These flows are generated by one source node to one destination.

TABLE III. Simulation parameters for Mobile Scenario

\begin{tabular}{|l|l|l|}
\hline No. & Parameters & Values \\
\hline 1 & Area & $1000 \mathrm{~m} \mathrm{X} 1000 \mathrm{~m}$ \\
\hline 2 & Transmission range & $250 \mathrm{~m}$ \\
\hline 3 & Pause time & $2 \mathrm{sec}$ \\
\hline 4 & Mobility & $20 \mathrm{~m} / \mathrm{sec}$ \\
\hline 5 & Simulation time & $400 \mathrm{sec}$ \\
\hline 6 & Mac layer & IEEE 802.11 \\
\hline 7 & Channel Capacity & $2 \mathrm{Mbits} / \mathrm{s}$ \\
\hline 8 & Application Layer & FTP, TELNET \\
\hline 9 & Transport layer & $\begin{array}{l}\text { Reno, Vegas, New Reno, } \\
\text { FL-TCP }\end{array}$ \\
\hline 10 & No. of connection (FTP) & $1,5,10$ and 20 flows \\
\hline 11 & $\begin{array}{l}\text { No of connection } \\
\text { (FTP/TELNET) }\end{array}$ & $\begin{array}{l}(1 / 1),(2 / 3),(5 / 5),(10 / 10) \\
\text { Flows }\end{array}$ \\
\hline 12 & No. of nodes & 50 \\
\hline 13 & Routing protocols & AODV \\
\hline 14 & Packet size & 1460 \\
\hline 15 & $\begin{array}{l}\text { Congestion Window } \\
\text { Size }\end{array}$ & 32 Packet \\
\hline
\end{tabular}

Following are the performance metrics considered in this study:

Throughput is the average number of bytes successfully received by the final destination per unit time.

Fairness index is how well the network delivers packets from the source to the destination. A network should demonstrate some degree of fairness to each traffic flow. The network should share the available bandwidth equally among competing flows. We use the following equation to compute the fairness index [17] to assign a fairness index to a set of throughputs $X=\left(x_{1}, x_{2}, \ldots, x_{n}\right)$.

$$
f(X)=\left(\sum_{i=1}^{n} x_{i}\right)^{2} / n \sum_{i=1}^{n} x_{i}^{2}
$$

Since throughput is a non-negative value, the fairness index will always lie between 0 and 1 . If all users receive equal throughput, the fairness index is 1 .

Packet loss percentage is the ratio of total bytes retransmitted to the total bytes transmitted. Packet loss percentage is also an important metric to be considered 9

\section{CONCLUSION}

In this paper, we have proposed a fuzzy-based inference system based on the factors of expected throughput and actual throughput. We call our protocol FL-TCP. FL-TCP dynamically adjusts the congestion window size. Our scheme does not rely on any explicit feedback from the network and requires only sender side modifications. Through extensive simulations we show that the number of concurrent flows significantly affects the TCP performance metrics. Our proposed scheme achieves the desired goals of performance improvement compared to other TCP variants: Vegas, Reno and New Reno. 


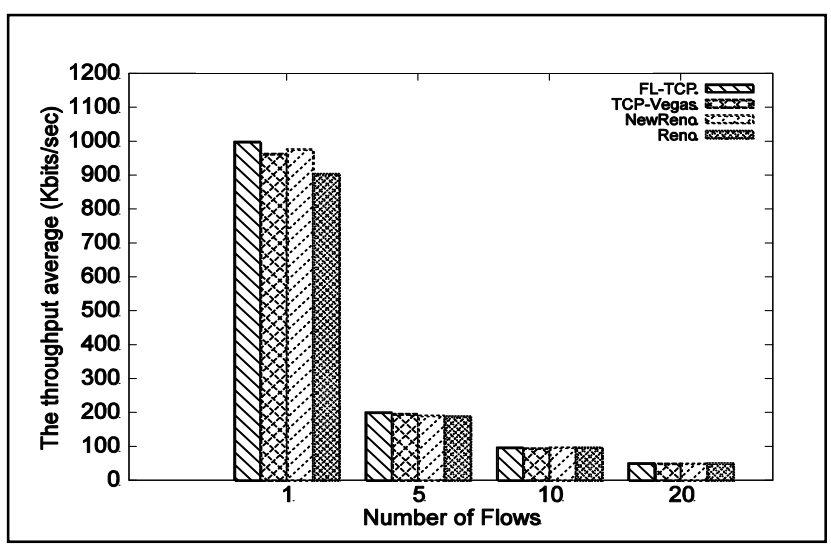

Fig 5: The Throughput Average (FTP Traffic Flows)

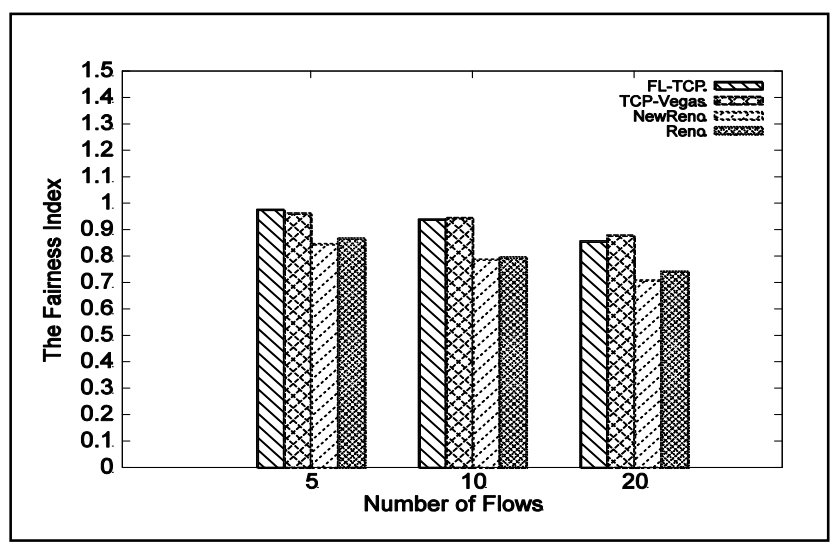

Fig 6: The Fairness Index (FTP Traffic Flows)

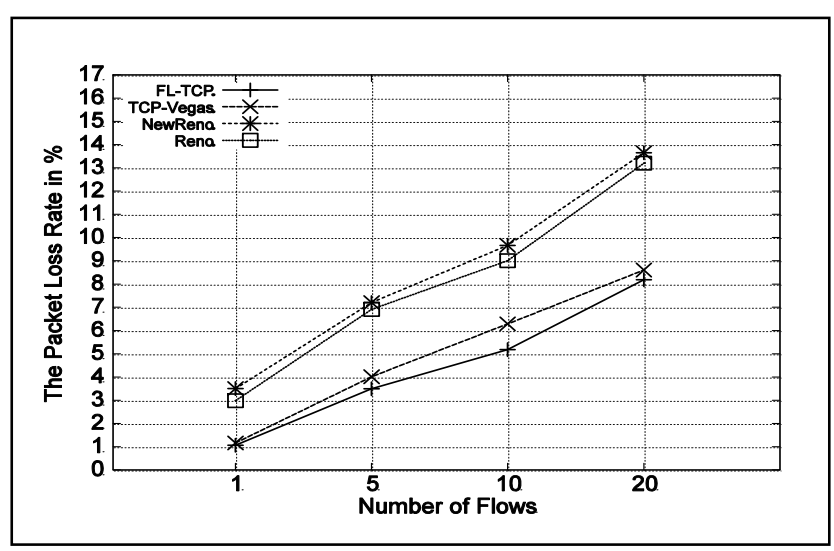

Fig 7: The packet loss rate in \% (FTP Traffic Flows)

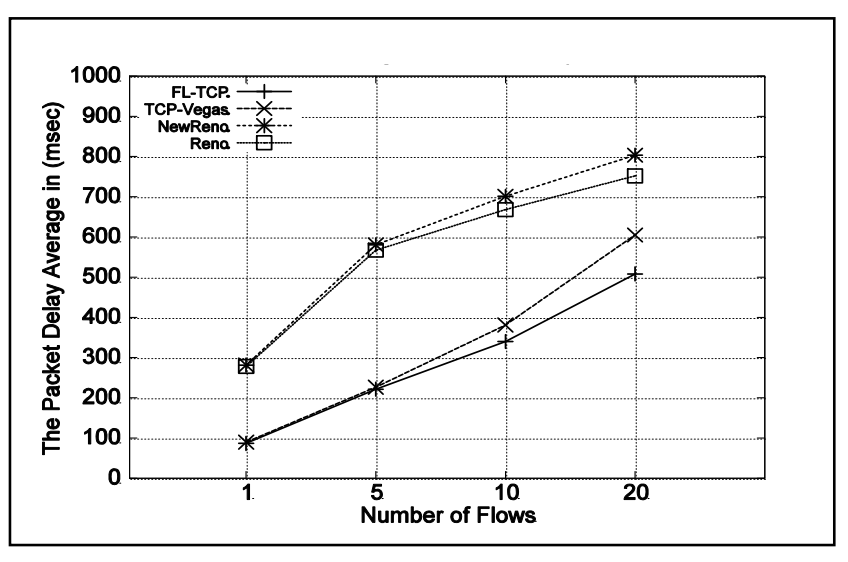

Fig 8: The packet delay average (FTP Traffic Flows)

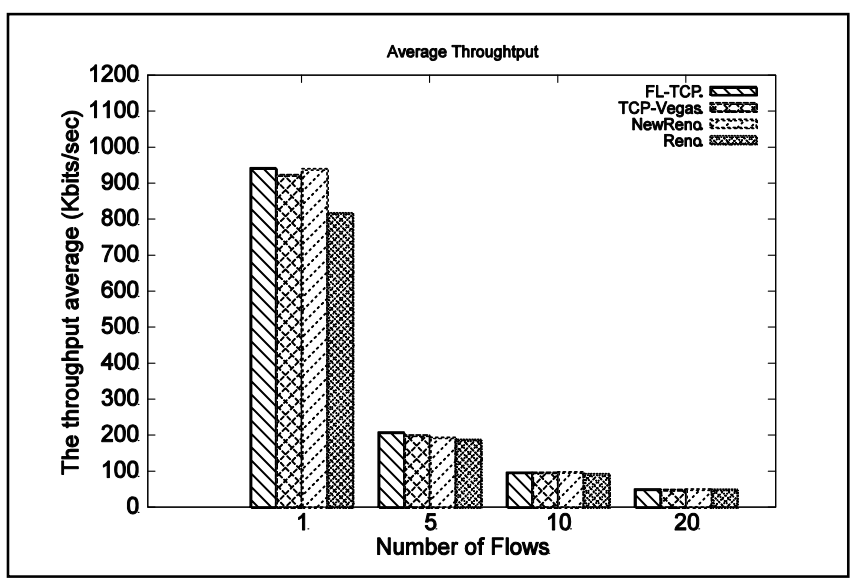

Fig 9: The throughput average (FTP/TELNET Traffic Flows)

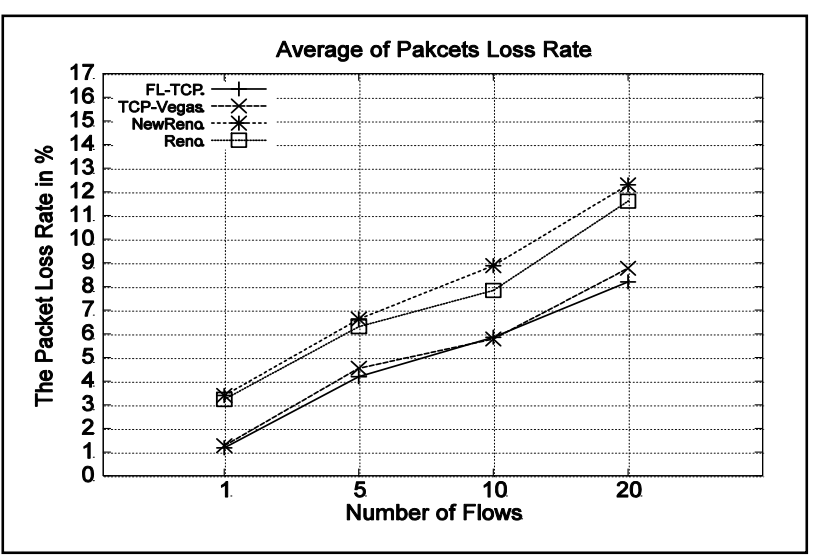

Fig 10: The fairness index (FTP/TELNET Traffic Flows) 


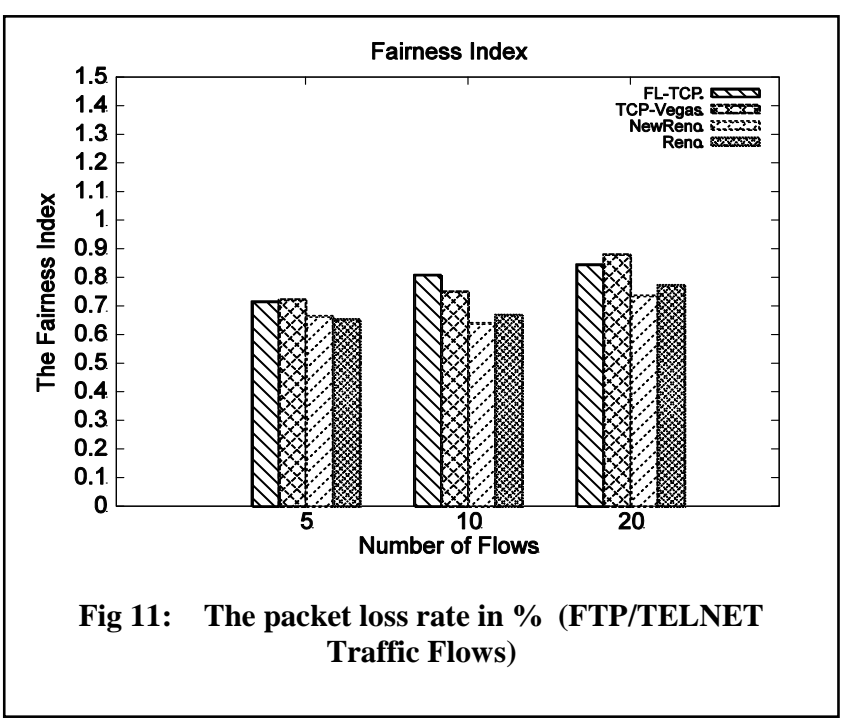

\section{REFERENCES}

[1] P.Ghosekar, G. Katkar and P. Ghorpade. 2010. Mobile Ad Hoc Networking: Imperatives and Challenges. In Proceeding of the IJCA Special Issue on Mobile Ad-hoc Networks.

[2] T. D. Dyer and R. V. Boppana. 2001. A Comparison of TCP Performance over Three Routing Protocols for Mobile Ad Hoc Networks. In Proceeding of ACM Symposium on Mobile Ad Hoc Networking \& Computing (Mobihoc), pp. 1-11, October 2001.

[3] G. Holland and N. Vaidya. 1999. Analysis of TCP over Mobile Ad Hoc Networks. In Proceeding of ACM MobiCom, pp. 219-230, August 1999.

[4] K. Chandran, S. Raghunathan, S. Venkatesan, and R. Prakash. 2001. A Feedback Based Scheme for improving TCP Performance in Ad Hoc Wireless Networks. IEEE Personal Communications Magazine, vol. 8,no. 1, pp. 3439, February 2001.

[5] J. Liu and S. Singh. 2001. ATCP: TCP for Mobile Ad Hoc Networks. IEEE Journal on Selected Area in Communications, vol. 19, no. 7, pp. 1300-1315, July 2001.

[6] K. Sundaresan, V. Anantharaman, H. Hsieh, and R. Sivakumar. 2003. ATP: A Reliable Transport Protocol for Ad-hoc Networks. In Proceeding of ACM MobiHoc, pp. 64-75, June 2003.

[7] N. C. Wang, Y. Huang, and W. Liu. 2008. A FuzzyBased Transport Protocol for Mobile Ad Hoc Networks. IEEE International Conference on Sensor Networks, Ubiquitous, and Trustworthy Computing, pp. 320-325, 2008. DOI 10.1109/SUTC.2008.52.

[8] R. Oliveira and T.Braun. 2004. A Delay-based Approach Using Fuzzy Logic to Improve TCP Error Detection in

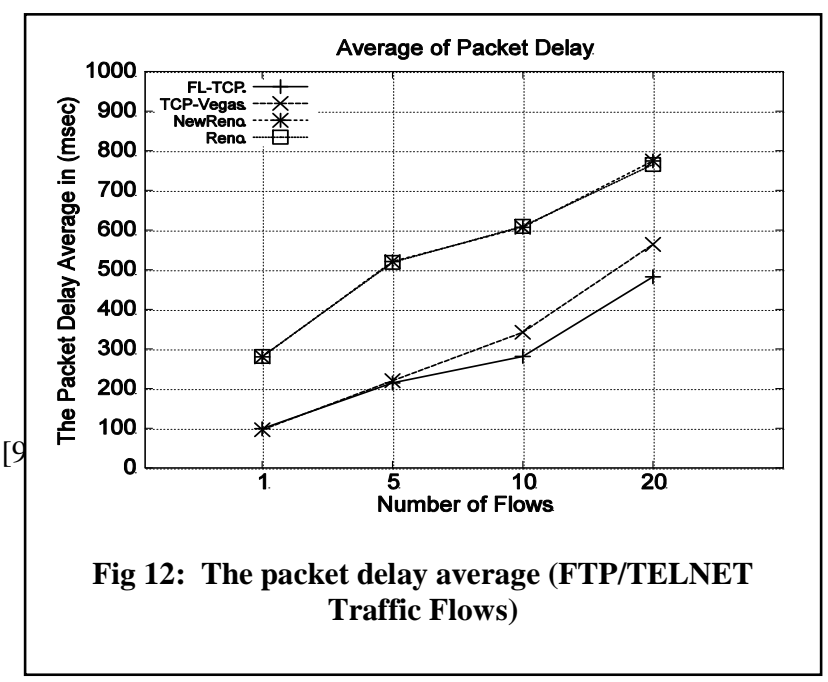

[10] Dmitri D. Perkins, Herman Hughes. 2002. Investigating the Performance of TCP in Mobile Ad Hoc Networks. Computer Communication, Vol. 25, pp. 1132-1139, 2002.

[11] M.Gerla, K. Tang, R. Bagrodia. 1999. TCP Performance in Wireless Multi-hop Networks. In Proceeding of IEEE WMCSA'99, New Orleans LA, Feb. 1999.

[12] D.D perkins, H. Hughes. 2000. A Performance Comparison of Routing Protocols for Mobile Ad hoc Networks. SPECTS 2000, Vancouver, BC. Canada, July 2000.

[13] K. T. Ibrahim, Nesar Ahmad, Salim Beg. 2011. A Congestion Window Control Mechanism Based on Fuzzy Logic to Improve TCP Performance in MANETs. In Proceeding of IEEE International Conference on Computational Intelligence and Communication Networks, IEEE CICN 2011, pp. 21-26, Gwalior, India. DOI: 10.1109/CICN.2011.5.

[14] C. Wang, Bo Li, K.Sohraby, and Y. Peng. 2003. AFRED: An Adaptive Fuzzy-based Control Algorithm for Active Queue Management. In Proceedings of the 28th Annual IEEE International Conference on Local Computer Networks (LCN'03), 2003.

[15] Jeonghoon M., Richard J. La, Venkat A. and W. Jean. 1999. Analysis and Comparison of TCP Reno and Vegas. In Proceeding of IEEE INFOCOM '99, 1999, pages 1556-1563, March 1999.

[16] K. Fall and K. V (editors). 2001. NS notes and documentation. The VINT Project, www.isi.edu/nsnam/ns/ns-documentation.html, April 2001.

[17] R. Jain. 1991. The Art of Computer System Performance Analysis, Wiley, New York, 1991. 